\title{
Monocyte/macrophage expression of ABCA1 has minimal contribution to plasma HDL levels
}

\author{
Mehrdad Haghpassand, Patricia-Ann K. Bourassa, Omar L. Francone, and Robert J. Aiello
}

Pfizer Global Research and Development, Groton, Connecticut, USA

Address correspondence to: Robert J. Aiello, Pfizer Global Research and Development, Department of Cardiovascular and Metabolic Diseases, Eastern Point Road, Groton, Connecticut 06340, USA.

Phone: (860) 441-6051; Fax: (860) 441-1128; E-mail: robert_j_aiello@groton.pfizer.com.

Received for publication March 26, 2001, and accepted in revised form September 4, 2001.

Excess accumulation of cholesterol in macrophages results in foam cell production and lesion development. Recent studies have demonstrated that ATP-binding cassette protein A1 (ABCA1) is highly regulated in macrophages and mediates the efflux of cholesterol and phospholipids to apolipoproteins, a process necessary for HDL formation. The goal of this study was to determine the contribution of monocyte/macrophage ABCA1 to HDL formation in vivo. We generated mice expressing $A B C A 1$ in macrophages and mice with selected inactivation of ABCA1 in macrophages by bone marrow transplantation in ABCA1-deficient $\left(A B C 1^{-/-}\right)$and wild-type (WT) mice. At all times, the level of $\mathrm{HDL}$ in $A B C 1^{-/-}$recipient mice remained low relative to WT recipient mice irrespective of the genotype of the donor macrophage ABCA1 or high-fat feeding. Expression of WT macrophage ABCA1 in $A B C 1^{-/-}$mice resulted in a small but significant increase in apoA-I levels starting 2 weeks after transplantation. No further increase in apoAI was observed up to 14 weeks after transplantation. The increase in apoAI was accompanied by a small but significant increase in HDL cholesterol 6 weeks after transplantation. The HDL formed as a consequence of the expression of WT macrophage ABCA1 migrated to the alpha position in a two-dimensional gel electrophoresis. These results demonstrate that monocyte/macrophage ABCA1 contributes to HDL formation; however, the contribution to the overall plasma HDL levels is minimal.

J. Clin. Invest. 108:1315-1320 (2001). DOI:10.1172/JCI200112810.

\section{Introduction}

Since the discovery of the inverse relationship between HDL levels and coronary artery disease, the structure, function, and metabolic origin of HDL has been a subject of intense investigation. HDL originates as discoidal complexes of apolipoproteins and phospholipids. Nascent HDL particles are either secreted by the liver (1) or the intestine (2) or assembled in the plasma from products of the catabolism of triglyceride-rich lipoproteins (3). Lipid-poor HDL precursors mature by obtaining cholesterol and phospholipids from cell membranes or triglyceride-rich lipoproteins. The removal of cellular cholesterol from peripheral tissues occurs by a number of mechanisms, including aqueous diffusion to a phospholipid-containing acceptor (4), facilitated efflux mediated by cell surface receptors such as SR-BI (5), as well as the release of membrane cholesterol to apolipoproteins by the action of a newly identified transporter, ATPbinding cassette $\mathrm{A} 1$ (ABCA1) (6).

Mutations in the ABCA1 transporter gene have been identified as the molecular defect responsible for the phenotype observed in Tangier disease (7-9). The pathophysiologic hallmarks of Tangier disease include orange tonsils, peripheral neuropathy, low levels of LDL cholesterol, and a virtual absence of apoAI and
HDL (10). More recently, studies in mice with targeted inactivation of ABCA1 (11) have demonstrated a critical role of ABCA1 in lipid metabolism and confirmed its role in the biogenesis of HDL. ABCA1 is expressed in many organs and is thought to mediate HDL maturation by facilitating the efflux of cellular phospholipids and cholesterol onto apolipoproteins. Interestingly, despite near-ubiquitous expression of ABCA1 (12), the accumulation of cholesterol observed in Tangier disease or ABCA1-knockout mice appears to be restricted primarily to macrophages $(10,11)$.

Macrophages in the human and mouse play an essential role in the initiation and progression of atherosclerosis (13). In vitro, macrophage ABCA1 mediates the removal of cholesterol to apolipoproteins in a doseand time-dependent manner (14). Also, cholesterol loading $(6,12)$ or treatment with cAMP analogues $(6$, 14) dramatically induces the expression of ABCA1 in macrophages. The ability of macrophages to modulate $\mathrm{ABCA} 1$ and their important role in atherosclerosis raise the intriguing question as to whether the efflux of lipids from macrophages significantly contributes to the systemic levels of HDL.

The purpose of this study was to examine the functional role of $A B C A 1$ in macrophages and to determine the contribution of this cell type to plasma HDL levels 
in vivo. We generated mice expressing ABCA1 in macrophages and mice with selected inactivation of ABCA1 in macrophages by bone marrow transplantation. Our findings demonstrate that macrophage ABCA1 mediates the biogenesis of mature HDL; however, this contribution is insufficient to restore HDL plasma levels or modulate the overall HDL pool.

\section{Methods}

Animals. ABCA1-knockout mice were created in DBA1lac/J background as described previously (11). Wild type (WT) DBA1lac/J mice were purchased from The Jackson Laboratories (Bar Harbor, Maine, USA). Mice were weaned at 4 weeks of age, and the study began when animals were between 10 and 12 weeks old. All mice were maintained on a 12-hour light/dark cycle and fed a chow diet. During the last 3 weeks before termination of the study, half of the animals were put on a high-fat diet containing $20 \%$ of calories as protein, $50 \%$ of calories as carbohydrate, $20 \%$ by weight lard, $1 \%$ by weight corn oil, and $0.15 \%$ by weight cholesterol.

Bone marrow transplantation. At 8-10 weeks of age, the recipient WT and $A B C 1^{-/-}$female mice were divided into two groups. Bone marrow was harvested from tibias of male WT and $A B C 1^{-/-}$mice and injected into lethally irradiated (10 Gy) recipient mice as described previously $(15,16)$. The resulting four groups of mice will be referred to as $\mathrm{WT} \rightarrow A B C 1^{-/-}, A B C 1^{-/-} \rightarrow A B C 1^{-/}$, $\mathrm{WT} \rightarrow \mathrm{WT}$, and $A B C 1^{-/-} \rightarrow \mathrm{WT}$ denoting donor bone marrow transplanted to $(\rightarrow)$ the recipient mouse. All mice that received bone marrow transplantation survived and appeared to be in good health during the first 11 weeks of the study. During the last 3 weeks of the study, of the four animals in each group on the high-fat diet, one $\mathrm{WT} \rightarrow A B C 1^{-/-}$, two $A B C 1^{-/-} \rightarrow A B C 1^{-/-}$, one $\mathrm{WT} \rightarrow \mathrm{WT}$, and two $A B C 1^{-/-} \rightarrow$ WT died. Also, one $A B C 1^{-/-} \rightarrow A B C 1^{-/-}$ mouse on the chow diet died during the last week of the study. The cause of death was not determined.

Peritoneal macrophages isolation and cholesterol efflux. Upon the termination of the study, mice on the chow diet ( $n=3$ for each group) were injected intraperitoneally with $1 \mathrm{ml}$ of sterile $6 \%$ casein, and peritoneal exudate cells were harvested after 4 days by washing the peritoneal cavity with HBSS (Life Technologies Inc., Rockville, Maryland, USA) supplemented with 1\% FBS. Peritoneal cells were washed once and resuspended in RPMI-1640 plus 10\% FBS plus 2 mM glutamine plus 40 $\mu \mathrm{g} / \mathrm{ml}$ gentamicin sulfate at approximately one million cells per milliliter and plated in 96-well Costar plates $(0.2 \mathrm{ml} /$ well; Corning-Costar Corp., Acton, Massachusetts, USA) to determine cholesterol efflux to apoAI. Also, cells were plated at approximately two million cells per well in Costar six-well plates for DNA isolation. To determine the cholesterol efflux, cells plated in 96-well plates were labeled for 48 hours with $15 \mu \mathrm{Ci} / \mathrm{ml}$ $\left[1,2-{ }^{3} \mathrm{H}\right]$ cholesterol (NEN Life Science Products Inc., Boston, Massachusetts, USA) and treated for an additional 18 hours in the presence or absence of $0.3 \mathrm{mM}$ 8-(4-chlorophenylthio) adenosine $3^{\prime}, 5^{\prime}$-cyclic mono- phosphate (cpt-cAMP). Subsequently, cells were washed, and $0.2 \mathrm{ml}$ of $20 \mu \mathrm{g} / \mathrm{ml}$ human apoAI in RPMI-1640 was added to each well (14). At various times, media from each treatment was filtered through a $0.45-\mu \mathrm{m}$ multiscreen plate (Millipore Corp., Bedford, Massachusetts, USA), and the corresponding cells were washed once with PBS and lysed in $0.2 \mathrm{~N} \mathrm{NaOH}$. The radioactivity in filtered media and cell lysates were determined in a Wallac 1450 Microbeta Plus liquid scintillation counter (Perkin Elmer Life Sciences Inc., Boston, Massachusetts, USA). Percentage of radiolabeled cholesterol released (percentage of efflux) was calculated as (cpm in media/cpm in media + cpm in cell lysate $) \times 100$. Calculations were also performed using cpm at time zero as the denominator with similar results.

DNA isolation, PCR and RNase protection assay. Peritoneal macrophages were lysed in DNAzol reagent (Life Technologies Inc.). Genomic DNA was isolated by spooling, and the DNA was resuspended in $8 \mathrm{mM}$ $\mathrm{NaOH}$. PCR amplification was performed using a PCR kit (Roche Molecular Systems, Branchburg, New Jersey, USA). WT $A B C A 1$ was detected using a forward primer in exon 19 at position 167,602 (Genbank reference no. AF287263), 5'TGGGAACTCCTGCTAAAAT3', and a reverse primer at position 168,352 in exon $20,5^{\prime}$ CCATGTGGTGTGTAGACA3', resulting in a 750-bp PCR fragment. The $A B C 1^{-/-}$construct was detected using a forward primer at position 162,967 in intron 14, 5'TTTCTCATAGGGTTGGTCA3', and a reverse primer in the targeted sequence, 5'TGCAATCCATCTTGTTCAAT3', resulting in a 420-bp fragment. RNase protection assay was performed using a 227-bp ABCA1 probe (between nucleotides 3,399 and 3,627 using Genbank sequence X75926 for mouse $A B C A 1$ ) and a 103-bp cyclophilin probe from Ambion Inc. (Austin, Texas, USA). The RNase protection assay was performed using a PharMingen (San Diego, California, USA) RNase protection kit according to the manufacturer's protocol. The protected fragment was run on a Novex QuickPoint nucleic acid separation system (Invitrogen Corp., Carlsbad, California, USA), and bands were visualized by exposing the plates to autoradiography or quantitated using a Strom 860 phosphorimager.

Plasma lipid and lipoprotein analysis. Mouse plasma was isolated from blood collected from the retro-orbital plexus. Cholesterol levels were determined using enzymatic colorimetric assays (Roche Molecular Biochemicals, Indianapolis, Indiana, USA). HDL was separated from non-HDL lipoproteins by dextran sulfate precipitation (17). An ELISA determined apoAI as described previously (17). The distribution of apoAI among HDL species were determined by two-dimensional gel electrophoresis as described previously (18) using a biotinylated rabbit polyclonal Ab (BIODESIGN International, Kennebunk, Maine, USA) and ${ }^{125}$ I-labeled streptavidin (Amersham Pharmacia Biotech, Piscataway, New Jersey, USA) to detect mouse apoAI.

Statistics. Statistical significance was determined by unpaired student's $t$ test using the StatView statistical 


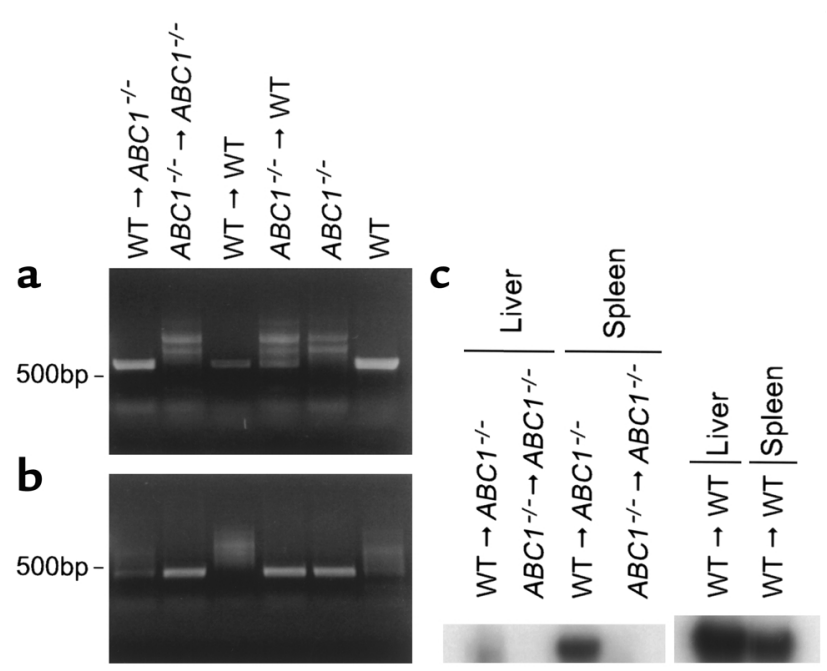

Figure 1

PCR amplification of $A B C A 1$ gene from WT and $A B C 1^{-/-}$peritoneal macrophages. Elicited peritoneal macrophages were obtained from irradiated mice transplanted with donor bone marrow (donor bone marrow $\rightarrow$ recipient mice). Total DNA was isolated and subjected to PCR amplification using specific primers for WT $A B C A 1$ (a) or the disrupted $A B C A 1$ (b) gene. RNase protection assay for $A B C A 1$ and cyclophilin was performed on $10 \mu \mathrm{g}$ total RNA obtained from the liver and spleen of recipient mice (c). Protected ABCA1 fragments were visualized by autoradiography and quantitated by a phosphorimager. Similar levels of cyclophilin were seen in all samples.

program (Abacus Concepts Inc., Berkeley, California, USA). Results are reported as mean plus or minus SD.

\section{Results}

Characterization of macrophages in recipient mice. To assess the contribution of macrophages to HDL synthesis, $A B C 1^{-/-}$mice were transplanted with bone marrow from WT mice. This resulted in mice that expressed ABCA1 in macrophages. Furthermore, mice with macrophage-specific inactivation of $A B C A 1$ were generated by transplanting bone marrow from $A B C 1^{-/-}$ mice in WT recipients. In addition, $A B C 1^{-/-} \rightarrow A B C 1^{-/-}$ and WT $\rightarrow$ WT transplantations were performed to generate the respective controls.

The repopulation of donor macrophages in the recipient mice was assessed by PCR analysis of elicited peritoneal macrophages 14 weeks after the bone marrow transplantation (Figure 1, a and b). Elicited peritoneal macrophages from $A B C 1^{-/-}$mice that received WT bone marrow, WT $\rightarrow A B C 1^{-1}$, contained DNA corresponding to WT $A B C A 1$, whereas their respective control, $A B C 1^{-/-} \rightarrow A B C 1^{-/}$, macrophages did not. Conversely, peritoneal macrophages from WT mice receiving $\mathrm{ABCA1}$-deficient bone marrow, $A B C 1^{-1-} \rightarrow \mathrm{WT}$, contained DNA corresponding to the $\mathrm{ABC1}^{-/-}$construct but the WT $\rightarrow$ WT control macrophages did not. Similar results were obtained when DNA from liver, lung, or spleen was used in the PCR analysis (data not shown). These results indicate the presence of donor macrophages in the irradiated recipient mice. To fur- ther ensure repopulation of tissue macrophages, the level of ABCA1 mRNA was determined in the liver and spleen from the recipient $A B C 1^{-/-}$mice by an RNase protection assay (Figure 1c). ABCA1 mRNA was detected in the liver and spleen of $A B C 1^{-/-}$mice repopulated with WT bone marrow but not in those transplanted with $A B C 1^{-/-}$bone marrow. Quantitatively, the level of ABCA1 mRNA in the liver and spleen of WT $\rightarrow A B C 1^{-/-}$mice were approximately $7 \%$ and $60 \%$ of the level of ABCA1 mRNA observed in the liver and spleen of WT mice, respectively. Assuming that Kupffer cells comprise about $20 \%$ of the liver, these results suggest approximately 35\% repopulation of liver macrophages from the donor bone marrow.

Functional activity of the ABCA1 transporter in the repopulated macrophages was assessed by the ability of pooled elicited peritoneal macrophages to mediate cholesterol efflux to human apoAI in response to cAMP analogues (14). As shown in Figure 2, a and c, macrophages isolated from mice transplanted with WT bone marrow exhibited WT response to a stable form of cAMP (cpt-cAMP). cpt-cAMP induced approximately a fivefold increase in apoAI-mediated efflux in both WT $\rightarrow A B C 1^{--}$and WT $\rightarrow$ WT macrophages. Interestingly, the basal level of efflux and ABCA1 mRNA levels (data not shown) were higher in $\mathrm{WT} \rightarrow A B C 1^{-1-}$ macrophages compared with WT $\rightarrow \mathrm{WT}$ macrophages. In contrast, macrophages isolated from mice that received $\mathrm{ABC1}^{-/-}$bone marrow (Figure 2, b and d) were completely unresponsive to cpt-cAMP irrespective of the genotype of the recipient mice. Taken together, the PCR analysis and the functional

a

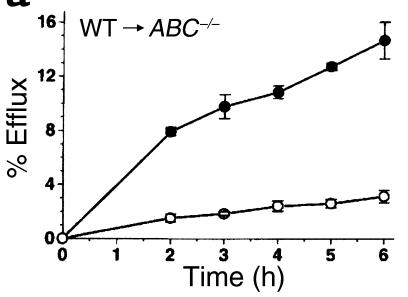

b
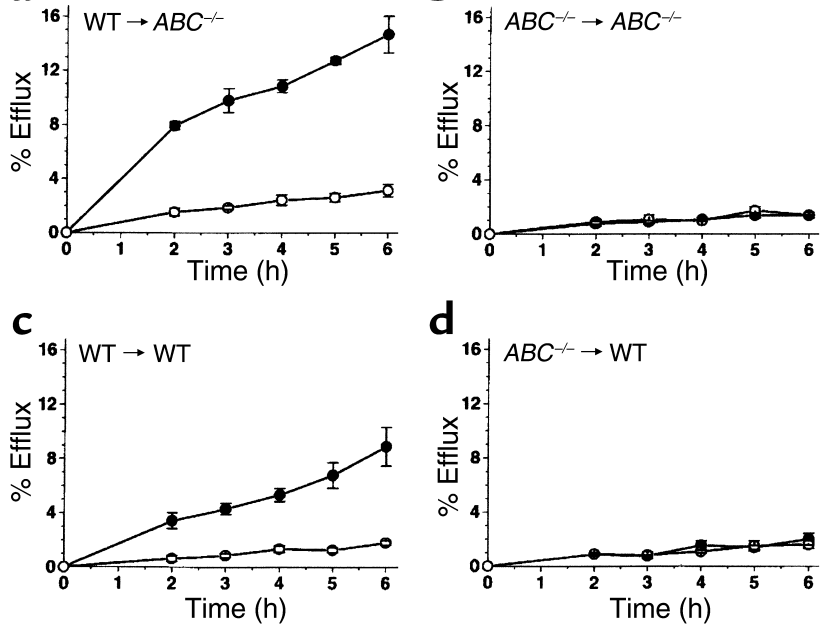

d

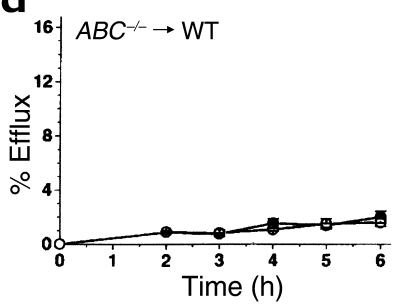

\section{Figure 2}

Cholesterol efflux from peritoneal macrophages in response to cptcAMP. Pooled peritoneal macrophages were obtained 14 weeks after transplantation. Cells were labeled with ${ }^{3} \mathrm{H}$-cholesterol for 48 hours and incubated for 18 hours in the presence (filled circles) or absence (open circles) of $0.3 \mathrm{mM}$ cpt-cAMP. Cholesterol efflux to $20 \mu \mathrm{g} / \mathrm{ml}$ of human apoAl was measured by determining the radioactivity in the media after various times as a percentage of total radioactivity in the cells and media $(n=3 \pm \mathrm{SE})$. 
Table 1

Plasma cholesterol and apoAl levels in mice after bone marrow transplantation.

\begin{tabular}{|c|c|c|c|c|c|c|c|c|c|c|c|c|c|}
\hline \multirow[b]{2}{*}{ Donor $\rightarrow$ recipient } & \multirow[b]{2}{*}{$n$} & \multicolumn{3}{|c|}{ Baseline } & \multicolumn{3}{|c|}{ Week 2} & \multicolumn{3}{|c|}{ Week 4} & \multicolumn{3}{|c|}{ Week 6} \\
\hline & & TPC & HDL-c ${ }^{A}$ & apoAIB & TPC & HDL-c & apoAl & TPC & HDL-c & apoAl & TPC & HDL-c & apoAl \\
\hline $\mathrm{WT} \rightarrow A B C 1^{-/-}$ & 8 & $\begin{array}{l}15.2 \\
(2.6)\end{array}$ & $\begin{array}{c}7.3 \\
(1.8)\end{array}$ & $\begin{array}{l}38.7 \\
(9.0)\end{array}$ & $\begin{array}{l}23.5 \\
(7.2)\end{array}$ & $\begin{array}{c}7.2 \\
(4.8)\end{array}$ & $\begin{array}{l}15.7^{c} \\
(2.3)\end{array}$ & $\begin{array}{l}19.1 \\
(9.1)\end{array}$ & $\begin{array}{c}5.6 \\
(1.0)\end{array}$ & $\begin{array}{l}10.0^{\mathrm{D}} \\
(2.8)\end{array}$ & $\begin{array}{l}28.1 \\
(4.5)\end{array}$ & $\begin{array}{l}3.95^{c} \\
(1.7)\end{array}$ & $\begin{array}{l}10.2^{\mathrm{C}} \\
(2.4)\end{array}$ \\
\hline$A B C 1^{-/-} \rightarrow A B C 1^{-/-}$ & 8 & $\begin{array}{c}17.4 \\
(3.6)\end{array}$ & $\begin{array}{c}7.5 \\
(1.3)\end{array}$ & $\begin{array}{l}42.7 \\
(9.1)\end{array}$ & $\begin{array}{l}23.8 \\
(7.4)\end{array}$ & $\begin{array}{c}4.3 \\
(3.6)\end{array}$ & $\begin{array}{c}10.1 \\
(2.6)\end{array}$ & $\begin{array}{l}31.6 \\
(8.3)\end{array}$ & $\begin{array}{c}4.7 \\
(1.0)\end{array}$ & $\begin{array}{c}7.2 \\
(1.0)\end{array}$ & $\begin{array}{l}28.1 \\
(4.7)\end{array}$ & $\begin{array}{c}1.6 \\
(1.2)\end{array}$ & $\begin{array}{c}6.2 \\
(1.2)\end{array}$ \\
\hline $\mathrm{WT} \rightarrow \mathrm{WT}$ & 7 & $\begin{array}{c}56.2 \\
(12.2)\end{array}$ & $\begin{array}{c}40.8 \\
(11.2)\end{array}$ & $\begin{array}{c}676.2 \\
(176.1)\end{array}$ & $\begin{array}{l}73.2 \\
(4.0)\end{array}$ & $\begin{array}{l}47.1 \\
(2.0)\end{array}$ & $\begin{array}{c}688.1 \\
(152.1)\end{array}$ & $\begin{array}{l}69.4 \\
(7.4)\end{array}$ & $\begin{array}{l}44.7 \\
(7.3)\end{array}$ & $\begin{array}{c}744.3 \\
(112.5)\end{array}$ & $\begin{array}{l}83.3 \\
(4.4)\end{array}$ & $\begin{array}{l}50.8 \\
(4.8)\end{array}$ & $\begin{array}{r}703.7 \\
(134.5\end{array}$ \\
\hline$A B C 1^{-/-} \rightarrow W T$ & 8 & $\begin{array}{l}58.3 \\
(7.2)\end{array}$ & $\begin{array}{l}36.7 \\
(2.5)\end{array}$ & $\begin{array}{c}706.8 \\
(118.8)\end{array}$ & $\begin{array}{c}73.9 \\
(11.9)\end{array}$ & $\begin{array}{l}44.8 \\
(3.8)\end{array}$ & $\begin{array}{c}710.4 \\
(159.5)\end{array}$ & $\begin{array}{c}77.0 \\
(12.0)\end{array}$ & $\begin{array}{l}46.1 \\
(5.0)\end{array}$ & $\begin{array}{l}781.3 \\
(87.4)\end{array}$ & $\begin{array}{c}85.3 \\
(11.8)\end{array}$ & $\begin{array}{l}45.9 \\
(5.7)\end{array}$ & $\begin{array}{l}696.9 \\
(86.7)\end{array}$ \\
\hline
\end{tabular}

Plasma samples were obtained from each mouse on chow diet 1 day before (baseline) and 2, 4, and 6 weeks after the transplantation by retro-orbital bleed ing. Total and HDL cholesterol were determined enzymatically, and mouse apoAl was determined by an ELISA. SDs are shown in parentheses. ${ }^{\mathrm{A}} \mathrm{mg} / \mathrm{dl}$. ${ }^{\mathrm{B}} \mu \mathrm{g} / \mathrm{ml}$ $C P<0.001$ compared with $A B C^{-/-} \rightarrow A B C 1^{-/-}$. $D P<0.05$ compared with $A B C^{-/-} \rightarrow A B C 1^{-1-}$.

efflux assay demonstrate complete repopulation of donor macrophages in the recipient mice.

Plasma lipid and lipoprotein analysis. Changes in total plasma cholesterol (TPC), HDL cholesterol (HDL-c), and apoAI levels were monitored for 6 weeks after the bone marrow transplantation. HDL-c and apoAI levels remained low in $A B C 1^{-/-}$recipient mice compared with the WT recipient mice throughout the study. Also, compared with the baseline, apoAI and HDL-c in $A B C 1^{-/-}$recipients gradually decreased after transplantation (Table 1). By 2 weeks after transplantation, an approximately $50 \%$ greater level of mouse apoAI was observed in WT $\rightarrow A B C 1^{-/}$mice compared with $A B C 1^{-/-} \rightarrow A B C 1^{-/-}$control mice. The greater level of apoAI in WT $\rightarrow A B C 1^{-/}$mice was maintained throughout the study. There was also a higher level of HDL-c in WT $\rightarrow A B C 1^{-/-}$compared with $A B C 1^{-/-} \rightarrow A B C 1^{-/-}$mice at 6 weeks after the transplantation $(3.95 \pm 1.7$ vs. $1.6 \pm 1.2 \mathrm{mg} / \mathrm{dl}$, respectively; $P<0.001)$. However, no differences in apoAI or HDL-c were observed in WT mice that received $A B \mathrm{C1}^{-/-}$bone marrow compared with WT $\rightarrow \mathrm{WT}$ controls at any time point. Also, no significant differences were observed in non-HDL cholesterol levels.

To determine the distribution of HDL subspecies in mice after transplantation, pooled plasma samples from each group were subjected to a two-dimensional gradient gel electrophoresis, and the distribution of apoAI among HDL species was determined by immunoblotting (Figure 3). The presence of WT macrophages in $A B C 1^{-/}$mice resulted in the appearance of a small population of $\alpha$-migrating HDL, accounting for the greater apoAI and HDL levels in plasma samples from these mice. No apparent difference was observed in WT recipient mice transplanted with WT or $A B C 1^{-/-}$bone marrow.

Response to high-fat diet. Several studies have demonstrated that ABCA1 expression is increased in macrophages loaded with cholesterol, resulting in greater efflux to free apoAI $(6,12)$. To determine if a high-fat and high-cholesterol diet increases the contribution of macrophage-derived cholesterol to HDL, four mice from each group were put on a Western-type, high-fat diet for 3 weeks before the termination of the study (between week 11 and week 14 after bone marrow transplantation). Subsequently, HDL-c and apoAI levels were determined at the end of the feeding period (Figure 4). High-fat feeding increased the HDL cholesterol and apoAI levels compared with chow diet in all animals; however, the HDL and apoAI levels in the $A B C 1^{-/-}$recipients remained low irrespective of the genotype of the donor bone marrow.

\section{Discussion}

Macrophages, unlike other peripheral cells, can accumulate high levels of cholesterol and lipids. Increased level of cholesterol in macrophages upregulates

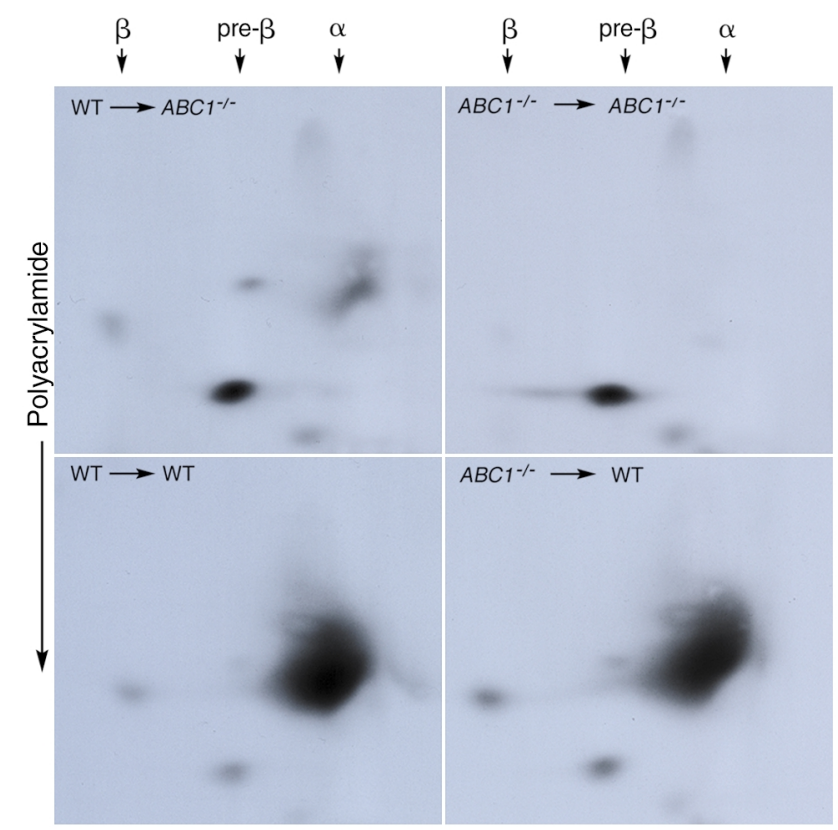

\section{Figure 3}

Distribution of mouse apoAl by two-dimensional gel electrophoresis. Fourteen weeks after transplantation, $15 \mu \mathrm{l}$ of pooled plasma from each transplant group was applied to electrophoreses in $0.75 \%$ agarose gel, which was then applied to a $3-16 \%$ polyacrylamide gradient gel. Plasma proteins were transferred to nitrocellulose membranes, and mouse apoAI was detected using a rabbit polyclonal $\mathrm{Ab}$. 

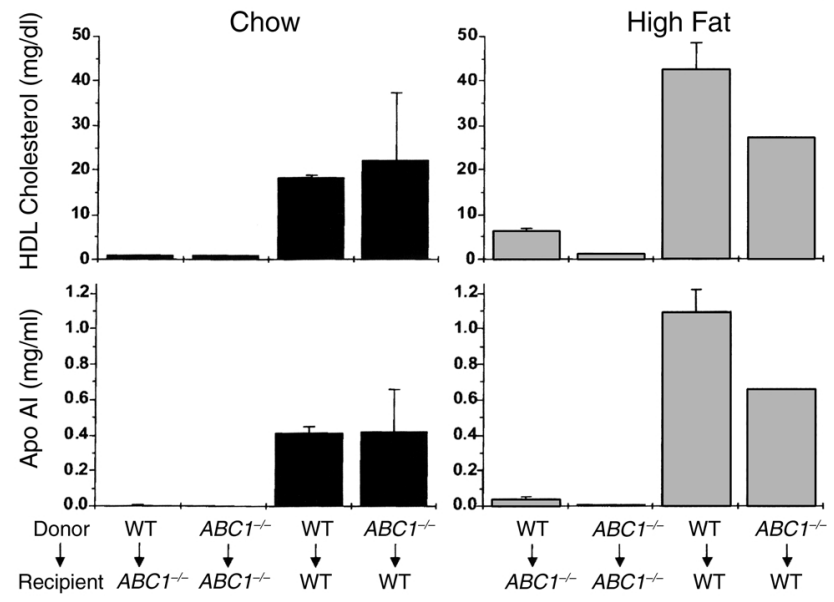

ABCA1, resulting in the removal of excess cholesterol to apolipoproteins. The critical role of ABCA1 in HDL formation has prompted speculation that pharmacological regulation of ABCA1 in macrophages can directly modulate HDL levels. Our results demonstrate that the repopulation of $A B C 1^{-/-}$mice with WT macrophages results in a minor increase in the steady state HDL levels up to 14 weeks after the transplantation. This study also suggests that steady state HDL levels represent the contribution from many tissues that express ABCA1 as opposed to only those with the greatest need for cholesterol efflux such as macrophages.

A conservative estimate for the total number of macrophages in a mouse is approximately $10^{8}$ cells (19). These cells comprise a very small portion of the total peripheral cells in the body. Although the exact level of cholesterol flux from macrophages has not been determined in vivo, the relative proportion of total cholesterol flux contributed by the macrophages must be small. This assumption is substantiated by the observation that tissues that have high level of resident macrophages, such as the lung and the spleen, have little contribution to the total cholesterol flux through the liver (20). It is therefore unlikely that a major part of cholesterol in HDL originates from the macrophages. However, macrophages may act as the initial donors of phospholipids and cholesterol to initiate the formation of mature HDL especially because the liver, where nascent HDL is formed from secreted apolipoproteins, contains the largest macrophage population in the body (approximately $20 \%$ of total body macrophages) (19). Once the mature HDL is formed, it can obtain cholesterol by other receptormediated pathways such as SR-BI (5) or from the lipolysis of remnant lipoproteins. Our results demonstrate that the contribution of macrophages to the formation of mature HDL is minimal. The expression of WT macrophages with functional ABCA1 in the $A B C 1^{-/-}$mice resulted in only a small increase in HDL. Nonetheless, the HDL formed as a consequence of normal ABCA1 expression by macrophages migrated

\section{Figure 4}

Comparison of plasma HDL cholesterol and apoAl levels in mice fed high-fat or chow diet 14 weeks after bone marrow transplantation. Three weeks before the termination of the study, transplanted mice were put on a chow or high-fat diet. Plasma HDL cholesterol and apoAl levels were determined. $n=3$ for all groups except $A B C 1^{-/-} \rightarrow A B C 1^{-/-}$and $A B C 1^{-/-} \rightarrow W T$ in the high-fat group, in which $n=2$.

to the alpha position on a two-dimensional gel, suggesting that macrophages do contribute to the formation of mature HDL but the level of contribution is very small. The formation of mature HDL is also dependent on plasma enzymes such as lecithin:cholesterol acyltransferase $(21,22)$ and phospholipid transfer protein (23). It is possible that these factors are markedly reduced in $A B C 1^{-/-}$mice, thus preventing the formation of large HDL. In our study, introducing ABCA1-deficient bone marrow to WT mice $\left(A B C 1^{-1-} \rightarrow\right.$ WT $)$ did not inhibit HDL formation, suggesting that in the presence of all of the factors necessary for mature HDL production, macrophage ABCA1 ablation has no major effect on the HDL level. (See results for $A B C 1^{-1-} \rightarrow \mathrm{WT}$ in Table 1). Also, highfat feeding, which induces foam cell formation and upregulates $\mathrm{ABCA} 1$, does not substantially increase HDL levels in $A B C 1^{-/}$mice transplanted with WT bone marrow up to 14 weeks after transplantation. Nevertheless, a study by Kennedy et al. has demonstrated slow repopulation of tissue macrophages after transplantation in mice (24). Based on the RNase protection study, we can estimate that approximately $7 \%$ of WT level of ABCA1 RNA is expressed in the liver from ABCA1-deficient mice. Considering that Kupffer cells constitute, at the most, $20 \%$ of an adult liver, these results suggest a 35\% repopulation of liver macrophages in ABCA1-deficient mice. These results were also confirmed by Y-chromosome quantitation in the recipient liver (data not shown).

Patients with Tangier disease or $A B C 1^{-/-}$mice do not have gross accumulation of lipid in their peripheral tissues. However, an abnormality in macrophage accumulation has been observed in patients with Tangier disease. Increased susceptibility to macrophage accumulation may also be responsible for moderately increased risk of coronary artery disease in these patients (10). Expression of ABCA1 in macrophages may have a beneficial role in atherosclerosis by facilitating cholesterol efflux and preventing foam cell production. Studies in apoE-knockout mice have demonstrated that small changes in plasma apoE as a consequence of apoE expression by macrophages have a profound effect on atherosclerosis $(15,25)$. Although the role of macrophage ABCA1 on atherosclerosis remains to be determined, the expression of ABCA1 in macrophages does not seem to be a major contributing factor to the heterogeneity observed in the plasma HDL levels. 


\section{Acknowledgments}

We thank Jeff McNamara and Kathy Murray at Charles River for their excellent technical and organizational help with bone marrow transplantation and animal husbandry. We also thank Lori Royer and Kenneth Hoppe for their assistance and Barrett Rollins for graciously allowing us to use the facilities at Dana Farber Cancer Institute for irradiation of mice.

1. Hamilton, R.L., Williams, M.C., Fielding, C.J., and Havel, R.J. 1976. Discoidal bilayer structures of nascent high density lipoproteins from perfused rat liver. J. Clin. Invest. 58:667-680.

2. Danielsen, E.M., Hansen, G.H., and Poulsen, M.D. 1993. Apical secretion of apolipoproteins from enterocytes. J. Cell Biol. 120:1347-1356.

3. Tall, A.R., Blum, C.B., Forester, G.P., and Nelson, C.A. 1982. Changes in the distribution and composition of plasma high density lipoproteins after ingestion of fat. J. Biol. Chem. 257:198-207.

4. Rothblat, G.H., et al. 1999. Cell cholesterol efflux: integration of old and new observations provides new insights. J. Lipid Res. 40:781-796.

5. Ji, Y., et al. 1997. Scavenger receptor BI promotes high density lipoprotein-mediated cellular cholesterol efflux. J. Biol. Chem. 272:20982-20985.

6. Lawn, R.M., et al. 1999. The Tangier disease gene product controls the cellular apolipoprotein-mediated lipid removal pathway. J. Clin. Invest. 104:R25-R31.

7. Brooks-Wilson, A., et al. 1999. Mutations in ABC1 in Tangier disease and familial high density lipoprotein deficiency. Nat. Genet. 22:336-345.

8. Bodzioch, M., et al. 1999. The gene encoding ATP binding cassette transporter 1 is mutated in Tangier disease. Nat. Genet. 22:347-351.

9. Rust, S., et al. Tangier disease is caused by mutations in the gene encoding ATP binding cassette transporter 1. Nat. Genet. 22:352-255.

10. Assmann, G., von Eckardstein, A., and Brewer, H.B., Jr. 1995. Familial high density lipoprotein deficiency: Tangier disease. In The metabolic and molecular bases of inherited disease. 7 th edition. C.A. Scriver, A.L. Beaudet, W.S. Sly, D. Valle, editors. New York, New York, USA. McGraw-Hill. 2053-2072.

11. McNeish, J., et al. 2000. High density lipoprotein deficiency and foam cell accumulation in mice with targeted disruption of ATP-binding cassette transporter-1. Proc. Natl. Acad. Sci. USA. 97:4245-4250.

12. Langmann, T., et al. 1999. Molecular cloning of the human ATP binding cassette transporter 1 (hABC1): evidence for sterol-dependent regu- lation in macrophages. Biochem. Biophys. Res. Commun. 257:29-33.

13. Ross, R. 1993. The pathogenesis of atherosclerosis: a perspective for the 1990s. Nature. 362: 801-809.

14. Bortnick, A.E., et al. 2000. The correlation of ATP-binding cassette 1 mRNA levels with cholesterol efflux from various cell lines. J. Biol. Chem. 275:28634-28640.

15. Boisvert, W.A., Spangenberg, J., and Curtiss, L.K. 1995. Treatment of severe hyperlipidemia in apolipoprotein E-deficient mice by bone marrow transplantation. J. Clin. Invest. 96:1118-1124.

16. Aiello, R.J., et al. 1999. Monocyte chemoattractant protein-1 accelerates atherosclerosis in apolipoprotein E-deficient mice. Arterioscler. Thromb. Vasc. Biol. 19:1518-1525.

17. Francone, O.L., Haghpassand, M., Bennett, J.A., Royer, L., and McNeish, J. 1997. Expression of human lecithin: cholesterol acyltransferase in transgenic mice: effects on cholesterol efflux, esterification and transport. J. Lipid Res. 38:813-822.

18. Francone, O.L, Royer., L., and Haghpassand, M. 1996. Increased pre $\beta$ HDL levels, cholesterol efflux, and LCAT-mediated esterification in mice expressing human cholesteryl ester transfer protein (CETP) and human apolipoprotein A-I (apo A-I) transgenes. J. Lipid Res. 37:1268-1277.

19. Lee, S.-H., Starkey, P.M., and Gordon, S. 1983. Quantitative analysis of total macrophage content in adult mouse tissues. Immunochemical studies with monoclonal antibody F4/80. J. Exp. Med. 161:475-489.

20. Jolley, C.D., Wollett, L.A., Turley, S.D., and Dietschy, J.M. 1998. Centripetal cholesterol flux to the liver is dictated by events in the peripheral organs and not by the plasma high density lipoprotein or apolipoprotein A-I concentration. J. Lipid Res. 39:2143-2149.

21. Fielding, C., and Fielding, P.E. 1995. Molecular physiology of reverse cholesterol transport. J. Lipid Res. 36:211-228.

22. Ng, D.S., Francone, O.L., Forte, T.M., Zhang, J., Haghpassand, M., and Rubin, E.M. 1997. Disruption of the murine lecithin:cholesterol acyltransferase gene causes impairment of adrenal lipid delivery and up-regulation of scavenger receptor class B type I. J. Biol. Chem. 272:15777-15781.

23. Jiang, X.C., et al. 1999. Targeted mutation of plasma phospholipid transfer protein markedly reduces high density lipoprotein levels. J. Clin. Invest. 103:907-914

24. Kennedy, D.W., and Abkowitz, J.L. 1997. Kinetics of central nervous system microglial and macrophage engraftment: analysis using a transgenic bone marrow transplantation model. Blood. 90:986-993.

25. Fazio, S., et al. 1997 Increased atherosclerosis in mice reconstituted with apolipoprotein E null macrophages. Proc. Natl. Acad. Sci. USA. 94:4647-4652. 